\title{
Efeito da facectomia no posicionamento palpebral superior: comparação entre as técnicas de facoemulsificação e a extracapsular
}

\author{
Changes in the position of the upper eyelid after cataract surgery:a comparison \\ between phacoemulsification and extracapsular techniques
}

\author{
Roberto Pinto Coelho ${ }^{1}$ \\ Raquel Nunes Paiva ${ }^{2}$ \\ Antonio Augusto Velasco e Cruz ${ }^{3}$
}

Trabalho realizado no Departamento de Oftalmologia, Otorrinolaringologia e Cirurgia de Cabeça e Pescoço da Faculdade de Medicina USP - Ribeirão Preto.

${ }^{1}$ Médico contratado do Hospital das Clínicas Universidade de São Paulo - Ribeirão Preto, Doutor em Oftalmologia pela Universidade de São Paulo - Ribeirão Preto; Diretor Clínico do Banco de Olhos do Hospital das Clínicas da Faculdade de Medicina USP - Ribeirão Preto.

${ }^{2}$ Doutora em Oftalmologia pela Universidade de São Paulo - Ribeirão Preto.

${ }^{3}$ Professor associado, responsável pelo setor de Plástica Ocular da Faculdade de Medicina USP - Ribeirão Preto.

Endereço para correspondência: Depto. Oftalmologia/Otorrinolaringologia/Cirurgia de Cabeça e Pescoço Av. Bandeirantes, 3900 - Ribeirão Preto (SP) CEP 14049-900 - E-mail: rpcoelho@keynet.com.br

Recebido para publicação em 30.04.2002 Aceito para publicação em 06.03.2003

Nota Editorial: Pela análise deste trabalho e por sua anuência na divulgação desta nota, agradecemos ao Dr. Eurípedes da Mota Moura.

\begin{tabular}{|l|}
\hline RESUMO \\
\hline Objetivo: Comparar o efeito de duas técnicas de facectomia (facoemulsi- \\
ficação e extracapsular) no posicionamento da pálpebra superior. Métodos: \\
Foi analisado o posicionamento palpebral de dois grupos de pacientes \\
submetidos à cirurgia de catarata: facoemulsificação e extracapsular. As \\
imagens foram digitalizadas (antes e 30 dias após a cirurgia) e processadas \\
com o programa NIH 1.62. O posicionamento palpebral foi medido em \\
relação a uma linha horizontal que unia os cantos medial e lateral da fenda \\
palpebral, passando pelo centro da pupila. Resultados: O posicionamento \\
palpebral foi afetado de maneira diferente segundo a técnica utilizada. Na \\
facoemulsificação a diferença média entre a posição palpebral superior pós \\
epré-operatória foi de-0,54 mm. Na extracapsular esta diferença aumentou \\
para -1,41 mm. O teste t de Student (amostras pareadas) mostrou que as \\
médias das diferenças entre os dois grupos são significativamente diferen- \\
tes (p=0,0068). Conclusão: A técnica de cirurgia de facoemulsificação \\
induziu menor variação do posicionamento palpebral em relação à cirurgia \\
extracapsular no pós-operatório recente.
\end{tabular}

Descritores: Blefaroptose/cirurgia; Extração de catarata/métodos; Facoemulsificação/ métodos; Pálpebras/cirurgia; Processamento de imagem assistida por computador/ métodos; Procedimentos cirúrgicos oftalmológicos/cirurgia

\section{INTRODUÇÃO}

A ocorrência de ptose palpebral após a cirurgia de catarata é bem conhecida. Em uma amostra de pacientes operados pela técnica extracapsular, Hosal et $\mathrm{al}^{(1)}$, demonstraram, em 6 meses de acompanhamento, uma prevalência de ptose de 7,3\%. Múltiplos fatores como o efeito miotóxico do anestésico local ${ }^{(2)}$, uso de blefarostato e de sutura fixadora do músculo reto superior $^{(3-4)}$ e tipo de anestesia ${ }^{(5-6)}$ já foram implicados na etiologia do abaixamento palpebral superior pós-operatório.

Nos últimos anos a facoemulsificação, vem se tornando a técnica de preferência para a cirurgia da catarata, pois essa técnica possibilita uma cirurgia mais rápida e menos traumática. De fato, se o tempo cirúrgico na técnica extracapsular é, em média, de 40 minutos, na facoemulsificação a duração do procedimento cai para 20 minutos. Além disso, na facoemulsificação a sutura de fixação do músculo reto superior não é usada e é possível o emprego de anestesia tópica ${ }^{(7-8)}$.

O objetivo do presente trabalho é comparar o efeito no posicionamento palpebral superior da cirurgia de catarata por facoemulsificação sob anestesia tópica e pela técnica extracapsular com anestesia peribulbar. 


\section{MÉTODOS}

A população de estudo consistiu de 42 pacientes, dos quais 22 foram operados por facoemulsificação sob anestesia tópica com colírio anestésico de cloridrato de proparacaína a $0,5 \%\left(\right.$ Anestalcon $\left.^{\circledR}\right)$ e sem sutura de fixação do músculo reto superior, e 20 operados pelo método extracapsular sob anestesia peribulbar de 4 mililitros de bupivacaína e 2 mililitros de lidocaína e com sutura de fixação do músculo reto superior.

Antes da cirurgia, os pacientes foram fotografados, com uma câmera fotográfica modelo Fotonex 4000SL Fujifilm, em posição primária do olhar, tomando-se o devido cuidado para minimizar eventuais rotações ântero-posteriores da cabeça. Outra foto era feita na ocasião da alta (1 mês de pós-operatório). Os dois grupos de fotos foram digitalizados e processados pelo programa NIH 1,62 que já foi utilizado em trabalho de quantificação da fenda palpebral ${ }^{(9)}$ (disponível pela internet no site do National Institute of Health (www.nimh.nih.gov). Inicialmente padronizou-se a angulação da fenda palpebral para zero desfazendo-se a inclinação entre o canto medial e o lateral. A seguir, traçava-se uma linha de base horizontal unindo o canto medial e o lateral. A partir dessa linha, o posicionamento palpebral era, então, medido com uma outra linha vertical passando necessariamente pelo centro da pupila até a margem palpebral superior (figura 1).

\section{RESULTADOS}

No grupo submetido à técnica extracapsular, a idade dos pacientes variou entre 44 e 87 anos (média de 65,4 anos). No grupo submetido à facoemulsificação a idade variou entre $59 \mathrm{e}$ 90 anos (média de 74 anos).

Em relação à duração da cirurgia, na técnica extracapsular variou de 35 a 95 minutos (média de 49,3 minutos), enquanto na facoemulsificação essa variação foi bem menor de 10 a 35 minutos (média de 17,68 minutos).

O posicionamento palpebral superior foi afetado de maneira diferente segundo a técnica utilizada. Na facoemulsificação, a diferença média entre o posicionamento pré e pós-operatório foi de - 0,54 mm, enquanto que na extracapsular, essa diferença

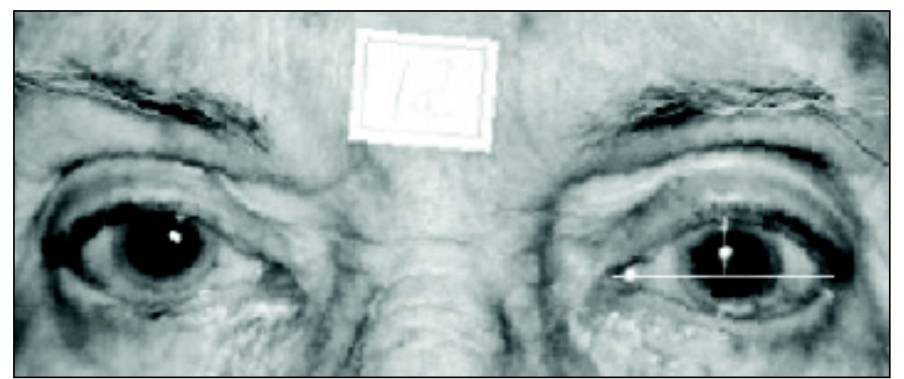

Figura 1 - Processamento de imagem para a mensuração do posicionamento palpebral superior (linha vertical). A etiqueta na região frontal era usada para a parametrização da imagem (elemento de medida conhecida para conversão pixel/milímetros) aumentou para $-1,41 \mathrm{~mm}$. O teste $t$ de Student mostrou que essas médias são significativamente diferentes $(\mathrm{p}=0,0068)$. A figura 2 compara as distribuições, nas duas técnicas, da variação do posicionamento palpebral. É fácil notar que com a extra, houve diferenças palpebrais de mais de $3,0 \mathrm{~mm}$, fato inexistente na facoemulsificação.

\section{DISCUSSÃO}

Com o avançar da idade, a aponeurose do músculo elevador da pálpebra pode sofrer um processo degenerativo que leva à atenuação ou mesmo desinserção dessa estrutura ${ }^{(10-13)}$. Esse processo, que é considerado uma conseqüência fisiológica do envelhecimento, pode se traduzir clinicamente por um abaixamento da margem palpebral superior ou manter-se subclínico, permitindo que a posição normal da margem superior seja mantida. Teoricamente, todos pacientes idosos que necessitam de cirurgia de catarata são candidatos à ptose aponeurótica.

No presente trabalho, verificou-se que independentemente da técnica utilizada, um discreto abaixamento da margem palpebral superior pode ser detectado, pelo menos, até 1 mês após a cirurgia. A variação da posição palpebral foi significantemente maior no grupo submetido à técnica extracapsular. No grupo operado pela faco essa variação foi, em média, negligenciável e praticamente subclínica, já que o efeito médio verificado $(0,54 \mathrm{~mm})$ é difícil de ser medida com régua milimetrada.

Vários fatores podem ser aventados para o maior efeito da técnica extracapsular sobre a posição da pálpebra superior. $\mathrm{Na}$ extracapsular, realiza-se a sutura de fixação do músculo reto superior, enquanto na facoemulsificação este procedimento não é necessário. $\mathrm{O}$ resultado das forças opostas criadas pelo blefarostato e a sutura de fixação do músculo reto superior podem causar a deiscência da aponeurose do músculo elevador da pálpebra devido à forte ligação entre o músculo reto superior e o elevador da pálpebra ${ }^{(14-15)}$. O próprio trauma cirúrgico no complexo reto/levantador da pálpebra durante a realização da sutura de fixação é considerado como um dos fatores
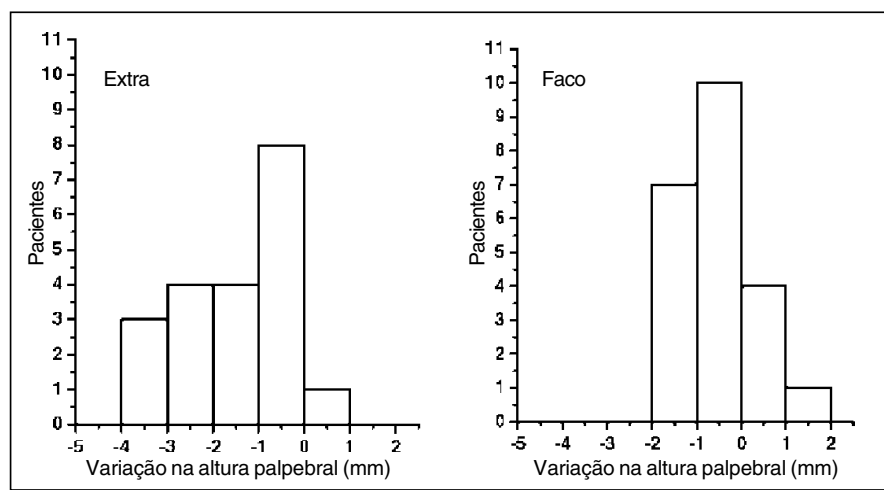

Figura 2 - Comparação entre as distribuições da variação da altura palpebral superior de pacientes submetidos a facectomia pela facoemulsificação e extracapsular 
mais importantes na etiologia da descida da pálpebra superior após a cirurgia de catarata ${ }^{(14)}$.

O grupo submetido à facoemulsificação recebeu anestesia tópica (colírio), enquanto o grupo submetido à extração da catarata pela técnica extracapsular recebeu anestesia peribulbar. A ptose palpebral após anestesia local já foi relatada como complicação de cirurgias oculares. A causa seria uma infiltração de anestésico inadvertidamente dentro do músculo elevador da pálpebra, sendo que esta ptose desapareceria espontaneamente após 8 a 12 semanas $^{(15)}$. Ptose de curta duração pode estar relacionada ao volume e à miotoxicidade da droga anestésica ${ }^{(2)}$. O grupo submetido à facoemulsificação não foi exposto à injeção de anestésico na área do complexo elevador/reto superior, o que pode explicar a menor variação da posição da pálpebra superior no pós-operatório recente em relação ao grupo submetido à extracapsular que recebeu um total de $6 \mathrm{ml}$ de anestésico pela via peribulbar.

A duração da cirurgia pela facoemulsificação foi praticamente a metade da duração da cirurgia pela técnica extracapsular. Desta forma o blefarostato permaneceu menos tempo nas pálpebras dos pacientes no primeiro grupo. Isto pode ocasionar um menor trauma no complexo elevador/reto superior, o que também pôde contribuir para uma menor variação da posição da pálpebra superior após a extração da catarata. Seja como for, os dados obtidos no presente trabalho sugerem fortemente que a ptose pós facectomia tende a desaparecer da prática oftalmológica com a melhoria da técnica cirúrgica possibilitada pela facoemulsificação.

Finalmente, deve-se lembrar que os dados coletados do presente trabalho referem-se a modificações do posicionamento palpebral ocorridas no pós-operatório recente (30 dias). Dados prospectivos de maior seguimento pós-operatório são necessários para se determinar o grau de transitoriedade dessas modificações.

\section{ABSTRACT}

Purpose: To compare changes in the upper eyelid position after cataract surgery with phacoemulsification and extracapsular techniques. Methods: The upper eyelid position was measured in two groups of patients before and 30 days after cataract surgery performed with phacoemulsification and extracapsular techniques. Palpebral fissure images (before and after the cataract surgery) were digitized and then processed using the NIH 1.62 software. Eyelid position was measu- red relative to a horizontal line passing through the pupil center and connecting both canthi of the palpebral fissure. Results: The palpebral position was differently affected according to the techniques. In phacoemulsification the mean difference between eyelids position before and after surgery was $-0.54 \mathrm{~mm}$. In the extracapsular technique this difference increased to $-1.41 \mathrm{~mm}$. The paired Student $\mathrm{t}$ test showed that the difference between two means was significant ( $\mathrm{p}=0.0068)$. Conclusion: The phacoemulsification technique induces less variation in the upper eyelid position than the extracapsular technique in the recent postoperative period.

Keywords: Blepharoptosis/surgery; Cataract extraction/methods; Phacoemulsification/methods; Eyelids/surgery; Image processing, computer-assisted/methods; Ophthalmologic surgical procedures/methods

\section{REFERÊNCIAS}

1. Hosal BM, Tekeli O, Gürsel E. Eyelid malpositions after cataract surgery. Eur J Ophthalmol 1998;8:12-5.

2. Rainin EA, Carlson BM. Postoperative diplopia and ptosis. A clinical hypothesis based on the myotoxicity of local anesthetics. Arch Ophtalmol 1985;103:1337-9.

3. Ropo A, Ruusuvaara P, Nikki P. Ptosis following periocular or general anaesthesia in cataract surgery. Acta Ophthalmol (Copenh). 1992; 70:262-5.

4. Kaplan LJ, Jaffe NS, Clayman HM. Ptosis and cataract surgery. A multivariant computer analysis of a prospective study. Ophthalmology 1985; 92:237-42.

5. Deady JP, Prince NJ, Sutton GA. Ptosis following cataract and trabeculectomy surgery. Br J Ophthalmol 1989;73:283-5

6. Feibel RM, Custer PL, Gordon MO. Postcataract ptosis. A randomized, double-masked comparison of peribulbar and retrobulbar anesthesia. Ophthalmology 1993;100:600-5

7. Novak KD, Koch DD. Topical anesthesia for phacoemulsification: initial 20 cases series whith one-month follow-up. J Cataract Refract Surg 1995;21:672-5.

8. Duguid IG, Claoué CM, Thamby-Rajah Y, Allan BD, Dart JK, Steele AD. Topical anaesthesia for phacoemulsification surgery. Eye 1995;9:456-9.

9. Cruz AAV, Baccega A. Análise bidimensional computadorizada da fenda palpebral. Arq Bras Oftalmol 2001;64:13-9.

10. Shore JW, McCord CD. Anatomic changes in involutional blepharoptosis. Am J Ophthalmol 1984;98:21-7.

11. Sutula FC. Histological changes in congenital and acquired blepharoptosis. Eye 1988;2:179-84.

12. Sanke RF. Relationship of senile ptosis to age. Ann Ophthalmol 1984; 16:928-31.

13. Singh SK, Sekhar GC, Gupta S. Etiology of ptosis after cataract surgery. J. Cataract Refract Surg 1997;23:1409-13.

14. Loeffler M, Solomon LD, Renaud M. Postcataract extraction ptosis: effect of the bridle suture. J Cataract Refract Surg 1990;16:501-4.

15. Rao VA, Kawatra VK. Ocular myotoxic effects of local anesthetics. Can J Ophthalmol 1988;23:171-3.

\section{Nos artigios enviados para publicação, o nome dos autores}

e suas afiliações devem estar completos. Isso facilitará a indexação e os links com as bases de dados e o CV Lates. 